\title{
On No-Node Solutions of the Lazer-McKenna Suspension Bridge Models
}

\section{Fanglei Wang, Kangbao Zhou}

College of Science, Hohai University, Nanjing, China

Email: wang-fanglei@hotmail.com, kangabaoz@sina.cn

Received 3 March 2015; accepted 23 June 2015; published 30 June 2015

\section{Abstract}

In this paper, we are concerned with the existence and multiplicity of no-node solutions of the Lazer-McKenna suspension bridge models by using the fixed point theorem in a cone.

\section{Keywords}

\section{Differential Equations, Periodic Solution, Cone, Fixed Point Theorem}

\section{Introduction}

In [1], the Lazer-McKenna suspension bridge models are proposed as following

$$
\left\{\begin{array}{c}
m_{1} v_{t t}-T v_{x x}+\delta_{2} v_{t}-k(u-v)^{+}=\varepsilon f_{1}(t, x), \\
m_{2} u_{t t}+E I u_{x x x x}+\delta_{1} u_{t}+k(u-v)^{+}=W_{1}(x), \\
u(0, T)=u(l . t)=u_{x x}(0, t)=u_{x x}(L, t)=0, \\
v(0, t)=v(L, T) \\
u(\bullet, x)=v(\bullet, x) \text { are } 2 \pi \text {-periodic in } \mathrm{t} .
\end{array}\right.
$$

If we look for no-node solutions of the form $u(x, t)=\lambda y(t) \sin (\pi x / L), \quad v(x, t)=\lambda z(t) \sin (\pi x / L)$ and impose a forcing term of the form $f_{i}(x, t, v, u)=\sin (\pi x / L) h_{i}(u, v)$, then via some computation, we can obtain the following system:

$$
\left\{\begin{array}{l}
y^{\prime \prime}+\delta_{1} y^{\prime}+a_{11}(t) y+a_{12}(t) z=\lambda h_{1}(y, z), \\
z^{\prime \prime}+\delta_{2} z^{\prime}+a_{21}(t) y+a_{22}(t) z=\lambda h_{2}(y, z), \\
y(t)=y(t+T), y^{\prime}(t)=y^{\prime}(t+T), z(t)=z(t+T), z^{\prime}(t)=z^{\prime}(t+T) .
\end{array}\right.
$$

In this paper, by combining the analysis of the sign of Green's functions for the linear damped equation, together with a famous fixed point theorem, we will obtain some existence results for (1) if the nonlinearities satisfy the following semipositone condition

(H) The function $h_{i}(y, z)$ is bounded below, and maybe change sign, namely, there exists a sufficiently large constant $\mathrm{M}>0$ such that $h_{i}(y, z)+M>0$. 
Such case is called as semipositone problems, see [2]. And one of the common techniques is the Krasnoselskii fixed point theorem on compression and expansion of cones.

Lemma 1.1 [3]. Let $E$ be a Banach space, and $K$ be a cone in $E$. Assume $\Omega_{1}, \Omega_{2}$ are open subsets of $E$ with $0 \in \Omega_{1}, \overline{\Omega_{1}} \subset \Omega_{2}$, Let $A: K \cap\left(\overline{\Omega_{2}} \backslash \Omega_{1}\right) \rightarrow K$ be a completely continuous operator such that either

(i) $\|A x\| \leq\|x\|, \forall x \in K \cap \partial \Omega_{1} ; \quad\|A x\| \geq\|x\|, \forall x \in K \cap \partial \Omega_{2}$; or

(ii) $\|A x\| \leq\|x\|, \forall x \in K \cap \partial \Omega_{2} ; \quad\|A x\| \geq\|x\|, \forall x \in K \cap \partial \Omega_{1}$;

Then, $A$ has a fixed point in $K \cap\left(\overline{\Omega_{2}} \backslash \Omega_{1}\right)$

\section{Preliminaries}

If the linear damped equation

$$
x^{\prime \prime}+h(t) x^{\prime}+a(t) x=0,
$$

is nonresonant, namely, its unique T-periodic solution is the trivial one, then as a consequence of Fredholm's alternative in [4], the nonhomogeneous equation $x^{\prime \prime}+h(t) x^{\prime}+a(t) x=e(t)$, admits a unique T-periodic solution which can be written as $x(t)=\int_{0}^{T} G(t, s) e(s) d s$, where $\mathrm{G}(\mathrm{t} ; \mathrm{s})$ is the Green's function of (2). For convenience, we will assume that the following standing hypothesis is satisfied throughout this paper:

(H1) $\delta_{i}(t), a_{i j}(t)$ are T-periodic functions such that the Green's function $G_{i}(t, s)$, associated with the linear damped equation

$$
x^{\prime \prime}+\delta_{i}(t) x^{\prime}+a_{i i}(t) x=0,
$$

is positive for all $(t, s) \in[0, T] \times[0, T]$, and $0<m_{i}=\min _{t, s \in[0, T]} G_{i}(t, s), M_{i}=\max _{t, s \in[0, T]} G_{i}(t, s)$.

(H2) $a_{12}(t), a_{121}(t)$ are negative T-periodic functions, and satisfy:

$$
a_{11}(t)+a_{12}(t)>0, a_{21}(t)+a_{22}(t)>0, M_{1}\left\|a_{12}(t)\right\|_{L^{1}} \leq \frac{1}{4}, M_{2}\left\|a_{21}(t)\right\|_{L^{1}} \leq \frac{1}{4} .
$$

Let $E$ denote the Banach space $C[0, T] \times C[0, T]$ with the norm $\|(y, z)\|=\max _{t \in[0, T]}|y(t)|+\max _{t \in[0, T]}|z(t)|$. for $(y, z) \in E$. Define $K$ to a cone in $E$ by $K=\{(y, z) \in E: y \geq 0, z \geq 0, y+z \geq \theta\|(y, z)\|\}$, where $\theta=\min _{i=1,2} \frac{m_{i}}{M_{i}}$. Also, for $r>0$ a positive number, let $K_{r}=\{(y, z) \in K:\|(y, z)\|<r\}, \quad \partial K_{r}=\{(y, z) \in K:\|(y, z)\|=r\}$.

If (H), (H1) and (H2) hold, let $\tilde{y}=y+\xi, \tilde{z}=z+\xi$, (1) is transformed into

$$
\left\{\begin{array}{c}
\tilde{y}^{\prime \prime}+\delta_{1} \tilde{y}^{\prime}+a_{11}(t) \tilde{y}+a_{12}(t) \tilde{z}=a_{11}(t) \xi+a_{12}(t) \xi+h_{1}(\tilde{y}-\xi, \tilde{z}-\xi), \\
\tilde{z}^{\prime \prime}+\delta_{2} \tilde{z}^{\prime}+a_{21}(t) \tilde{y}+a_{22}(t) \tilde{z}=a_{21}(t) \xi+a_{22}(t) \xi+h_{2}(\tilde{y}-\xi, \tilde{z}-\xi),
\end{array}\right.
$$

where $\xi$ is chosen such that

$$
\begin{aligned}
& a_{11}(t) \xi+a_{12}(t) \xi+\lambda h_{1}(\tilde{y}-\xi, \tilde{z}-\xi)>1, \\
& a_{21}(t) \xi+a_{22}(t) \xi+\lambda h_{2}(\tilde{y}-\xi, \tilde{z}-\xi)>1 .
\end{aligned}
$$

Let $B: K \rightarrow E$ be a map, which defined by $B(\tilde{y}, \tilde{z})(t)=\left(B_{1}(\tilde{y}, \tilde{z})(t), B_{2}(\tilde{y}, \tilde{z})(t)\right)$, where

$$
\begin{gathered}
B_{1}(\tilde{y}, \tilde{z})(t)=\int_{0}^{T} G_{1}(t, s)\left[-a_{12}(s) z(s)+F_{1}(\tilde{y}(s), \tilde{z}(s))\right] d s, \\
B_{2}(\tilde{y}, \tilde{z})(t)=\int_{0}^{T} G_{2}(t, s)\left[-a_{21}(s) y(s)+F_{2}(\tilde{y}(s), \tilde{z}(s))\right] d s, \\
F_{1}(\tilde{y}(s), \tilde{z}(s))=a_{11}(t) \xi+a_{12}(t) \xi+\lambda h_{1}(\tilde{y}-\xi, \tilde{z}-\xi), \\
F_{2}(\tilde{y}(s), \tilde{z}(s))=a_{21}(t) \xi+a_{22}(t) \xi+\lambda h_{2}(\tilde{y}-\xi, \tilde{z}-\xi) .
\end{gathered}
$$

$t$ is straightforward to verify that the solution of (1) is equivalent to the fixed point Equation $B(\tilde{y}, \tilde{z})(t)=(\tilde{y}(t), \tilde{z}(t))$. 
Lemma 2.1 Assume that (H), (H1) and (H2) hold. Then $B: K \rightarrow K$ is compact and continuous.

For convenience, define $h_{i, \infty}=\lim _{y+z \rightarrow+\infty} \frac{h_{i}(y, z)}{y+z}$, for any $y, z>0$.

Lemma 2.2 [2] Assume that (H), (H1) and (H2) hold. If $h_{i, \infty}=0$, then, for $\mathrm{i}=1$, 2, the functions $F_{i}$ are continuous on $R^{+} \times R^{+}, F_{i}(\tilde{y}(s), \tilde{z}(s))>1$ for $(\tilde{y}(s), \tilde{z}(s)) \in R^{+} \times R^{+}$, and $\lim _{\tilde{y}+\tilde{z} \rightarrow+\infty} \frac{F_{i}(\tilde{y}, \tilde{z})}{\tilde{y}+\tilde{z}}=0$.

Lemma 2.3 [2] Assume that (H), (H1) and (H2) hold. If $h_{i, \infty}=+\infty$, then, for $i=1$, 2, the functions $F_{i}$ are continuous on $R^{+} \times R^{+}, F_{i}(\tilde{y}(s), \tilde{z}(s))>1$ for $(\tilde{y}(s), \tilde{z}(s)) \in R^{+} \times R^{+}$, and $\lim _{\tilde{y}+\tilde{z} \rightarrow+\infty} \frac{F_{i}(\tilde{y}, \tilde{z})}{\tilde{y}+\tilde{z}}=+\infty$.

\section{Main Results}

Theorem 3.1 Assume that (H), (H1) and (H2) hold.

(I) Then there exists a $\lambda^{*}>0$ such that (1) has a positive periodic solution for $0<\lambda<\lambda^{*}$;

(II) If $h_{i, \infty}=0$, then for an $\lambda>0$, (1) has a positive periodic solution;

(III) If $h_{i, \infty}=+\infty$, then (1) has two positive periodic solutions for all sufficiently small $\lambda$.

Proof. (I) On one hand, take R $>0$ such that

$$
\xi \cdot \max \left\{M_{1}, M_{2}\right\} \cdot \max \left\{\left\|a_{11}\right\|_{L^{1}}+\left\|a_{12}\right\|_{L^{1}},\left\|a_{21}\right\|_{L^{1}}+\left\|a_{22}\right\|_{L^{1}}\right\}<\frac{R}{8} .
$$

Set $\Psi_{i}(R)=\max \left\{h_{i}(\tilde{y}-\xi, \tilde{z}-\xi): \theta R \leq\|(\tilde{y}, \tilde{z})\| \leq R\right\}$. Then, for each $(\tilde{y}, \tilde{z}) \in \partial K_{R}$, we have

$$
\begin{aligned}
\max _{t \in[0, T]} B_{1}(\tilde{y}, \tilde{z})(t) & =\max _{t \in[0, T]} \int_{0}^{T} G_{1}(t, s)\left[-a_{12}(s) z(s)+F_{1}(\tilde{y}(s), \tilde{z}(s))\right] d s, \\
& \leq M_{1}\left\|a_{12}\right\|_{L^{1}} \cdot\|(\tilde{y}, \tilde{z})\|+\lambda M_{1} \Psi_{1}(R)+M_{1}\left\|a_{11}\right\|_{L^{1}} \xi+M_{1}\left\|a_{12}\right\|_{L^{1}} \xi \\
& \leq \frac{R}{4}+\frac{R}{8}+\lambda M_{1} \Psi_{1}(R) .
\end{aligned}
$$

Then from the above inequalities, it follows that there exists a $\lambda_{1}^{*}>0$ such that

$$
\lambda M_{1} \Psi_{1}(R) \leq \frac{R}{8} \text {, for } 0<\lambda<\lambda_{1}^{*} .
$$

Furthermore, for any $(\tilde{y}, \tilde{z}) \in \partial K_{R}$, we obtain $\max _{t \in[0, T]} B_{1}(\tilde{y}, \tilde{z})(t) \leq \frac{R}{2}=\frac{\|(\tilde{y}, \tilde{z})\|}{2}$.

In the similar way, there exists a $\lambda_{2}^{*}>0$, such that $\lambda M_{2} \Psi_{2}(R) \leq \frac{R}{8}$, for $0<\lambda<\lambda_{2}^{*}$. and we also have

$$
\max _{t \in[0, T]} B_{2}(\tilde{y}, \tilde{z})(t) \leq \frac{R}{2}=\frac{\|(\tilde{y}, \tilde{z})\|}{2}, \text { for }(\tilde{y}, \tilde{z}) \in \partial K_{R} .
$$

So let us choose $\lambda=\min \left\{\lambda_{1}^{*}, \lambda_{2}^{*}\right\}$, and we can obtain

$$
\|B(\tilde{y}, \tilde{z})\|<\frac{R}{2}+\frac{R}{2}=\|(\tilde{y}, \tilde{z})\|, \text { for any }(\tilde{y}, \tilde{z}) \in \partial K_{R}, 0<\lambda<\lambda^{*} .
$$

On the other hand, from the condition $F_{i}(\tilde{y}(s), \tilde{z}(s))>1$ for all $(\tilde{y}(s), \tilde{z}(s)) \in R^{+} \times R^{+}$, it follows that there is a sufficient small $r>0$ such that $F_{i}(\tilde{y}, \tilde{z}) \geq \eta(\tilde{y}+\tilde{z})$ for $(\tilde{y}(s), \tilde{z}(s)) \in R^{+} \times R^{+}$, and $\tilde{y}(s)+\tilde{z}(s) \leq r$, where $\eta$ is chosen such that $\eta \cdot \min \left\{m_{1}, m_{2}\right\}>\frac{1}{2}$.

Then, for any $(\tilde{y}, \tilde{z}) \in \partial K_{r}$, we obtain

$$
B_{1}(\tilde{y}, \tilde{z})(t)=\int_{0}^{T} G_{1}(t, s)\left[-a_{12}(s) z(s)+F_{1}(\tilde{y}(s), \tilde{z}(s))\right] d s \geq \theta \eta m_{1}\|(\tilde{y}, \tilde{z})\|>\frac{\|(\tilde{y}, \tilde{z})\|}{2}
$$




$$
B_{2}(\tilde{y}, \tilde{z})(t)=\int_{0}^{T} G_{2}(t, s)\left[-a_{21}(s) y(s)+F_{2}(\tilde{y}(s), \tilde{z}(s))\right] d s \geq \theta \eta m_{2}\|(\tilde{y}, \tilde{z})\|>\frac{\|(\tilde{y}, \tilde{z})\|}{2} .
$$

So we have $\|B(\tilde{y}, \tilde{z})\|>\|(\tilde{y}, \tilde{z})\|$, for any $(\tilde{y}, \tilde{z}) \in \partial K_{r}$.

Therefore, from Lemma 1.1, it follows that the operator B has at least one fixed point $(\tilde{y}, \tilde{z})$ in $\bar{K}_{R} \backslash K_{r}$, for $0<\lambda<\lambda^{*}$.

(II) Since $h_{i, \infty}=0$, then from Lemma 2.1, it follows that $\lim _{\tilde{y}+\tilde{z} \rightarrow+\infty} \frac{F_{i}(\tilde{y}, \tilde{z})}{\tilde{y}+\tilde{z}}=0$. Define a function $\tilde{F}_{i \lambda}: R^{+} \rightarrow R^{+}$ as $\tilde{F}_{i \lambda}(s)=\max _{\tilde{y}+\tilde{z}<s} F_{i}(\tilde{y}, \tilde{z})$. By Lemma 2.5 in [2], it is easy to see that $\lim _{s \rightarrow+\infty} \frac{\tilde{F}_{i \lambda}(s)}{s}=0$. Thus by the definition, there is an $\bar{R}>2 r$ such that $\tilde{F}_{i \lambda}(\bar{R}) \leq \varepsilon \bar{R}$, where $\varepsilon$ satisfying $\varepsilon T \max \left\{M_{1}, M_{2}\right\} \leq \frac{1}{4}$.

Then, for each $(\tilde{y}, \tilde{z}) \in \partial K_{\bar{R}}$, we have

$$
\begin{aligned}
\max _{t \in[0, T]} B_{1}(\tilde{y}, \tilde{z})(t) & =\max _{t \in[0, T]} \int_{0}^{T} G_{1}(t, s)\left[-a_{12}(s) z(s)+F_{1}(\tilde{y}(s), \tilde{z}(s))\right] d s, \\
& \leq M_{1}\left\|a_{12}\right\|_{L^{1}} \cdot\|(\tilde{y}, \tilde{z})\|+\varepsilon M_{1} T\|(\tilde{y}, \tilde{z})\|<\frac{\|(\tilde{y}, \tilde{z})\|}{2} .
\end{aligned}
$$

In the similar way, for any $(\tilde{y}, \tilde{z}) \in \partial K_{R}$, we also have $\max _{t \in[0, T]} B_{2}(\tilde{y}, \tilde{z})(t)<\frac{\|(\tilde{y}, \tilde{z})\|}{2}$. Furthermore, from The above inequalities, we get $\|B(\tilde{y}, \tilde{z})\|<\|(\tilde{y}, \tilde{z})\|$, for any $(\tilde{y}, \tilde{z}) \in \partial K_{\bar{R}}$.

Therefore, from Lemma 1.1, it follows that B has one fixed point $(\tilde{y}, \tilde{z})$ in $\bar{K}_{\bar{R}} \backslash K_{r}$ for any $\lambda>0$.

(III) Since $h_{i, \infty}=+\infty$, then from Lemma 2.2, it follows that $\lim _{\tilde{y}+\tilde{z} \rightarrow+\infty} \frac{F_{i}(\tilde{y}, \tilde{z})}{\tilde{y}+\tilde{z}}=+\infty$. By the definition, there exists $R^{\prime}>0$, such that $F_{i}(\tilde{y}, \tilde{z}) \geq \vartheta(\tilde{y}+\tilde{z})$, where $\vartheta$ is chosen such that $\vartheta \theta T \min \left\{m_{1}, m_{2}\right\}>\frac{1}{2}$.

Choosing $\hat{R}=\max \left\{R+1, \frac{R^{\prime}}{\theta}\right\}$, and for any $(\tilde{y}, \tilde{z}) \in \partial K_{\tilde{R}}$, we have $\tilde{y}+\tilde{z} \geq \theta\|(\tilde{y}, \tilde{z})\|>R^{\prime}$ and

$$
\begin{aligned}
& B_{1}(\tilde{y}, \tilde{z})(t)=\int_{0}^{T} G_{1}(t, s)\left[-a_{12}(s) z(s)+F_{1}(\tilde{y}(s), \tilde{z}(s))\right] d s \geq \theta \vartheta m_{1} T\|(\tilde{y}, \tilde{z})\|>\frac{\|(\tilde{y}, \tilde{z})\|}{2}, \\
& B_{2}(\tilde{y}, \tilde{z})(t)=\int_{0}^{T} G_{2}(t, s)\left[-a_{21}(s) z(s)+F_{2}(\tilde{y}(s), \tilde{z}(s))\right] d s \geq \theta \vartheta m_{2} T\|(\tilde{y}, \tilde{z})\|>\frac{\|(\tilde{y}, \tilde{z})\|}{2} .
\end{aligned}
$$

Thus from the above inequalities, we can get $\|B(\tilde{y}, \tilde{z})\|>\|(\tilde{y}, \tilde{z})\|$, for any $(\tilde{y}, \tilde{z}) \in \partial K_{\hat{R}}$.

Therefore, from Lemma 1.1, it follows that the operator B has at least two fixed points $\left(\tilde{y}_{1}, \tilde{z}_{1}\right)$ in $\bar{K}_{\bar{R}} \backslash K_{r}$ and $\left(\tilde{y}_{2}, \tilde{z}_{2}\right)$ in $\bar{K}_{\hat{R}} \backslash K_{R}$. Namely, system (1) has two solutions for sufficiently small $\lambda>0$.

\section{References}

[1] Lazer, A.C. and McKenna, P.J. (1990) Large-Amplitude Periodic Oscillations in Suspension Bridges: Some New Connections with Nonlinear Analysis. Siam Review, 32, 537-578. http://dx.doi.org/10.1137/1032120

[2] Wang, H. (2009) Periodic Solutions to Non-Autonomous Second-Order Systems. Nonlinear Analysis: Theory, Methods \& Applications, 71, 1271-1275. http://dx.doi.org/10.1016/j.na.2008.11.079

[3] Dajun, G. and Lakshmikantham, V. (1988) Nonlinear Problems in Abstract Cones.

[4] Chu, J., Fan, N. and Torres, P.J. (2012) Periodic Solutions for Second Order Singular Damped Differential Equations. Journal of Mathematical Analysis and Applications, 388, 665-675. http://dx.doi.org/10.1016/j.jmaa.2011.09.061 